\title{
Strengthening Double Curved Arch Bridges by Using Extrados
}

\section{Section Augmentation Method}

\author{
Juntao Kang ${ }^{1, ~ a ~, ~ X u e f e n g ~ W a n g ~}{ }^{1, b}$, Jian Yang ${ }^{2, c}$, Yingang Du ${ }^{3, d}$ \\ ${ }^{1}$ Institute of Civil Engineering and Architecture, Wuhan University of Technology, \\ Wuhan, Hubei, 430070, China \\ ${ }^{2}$ School of Civil Engineering, University of Birmingham, Edgbaston, Birmingham, \\ B15 2TT, UK \\ ajtkang@163.com, bjakcn68@163.com, cj.yang.3@bham.ac.uk, \\ dYingang.du@anglia.ac.uk
}

\begin{abstract}
Double curved arch bridges have played an important role in the history of bridge constructions in China. The strengthening or retrofitting of this type of bridge has attracted a great deal of research attentions in the Chinese bridge engineering community. In this paper, a novel strengthening method, i.e. the extrados section augmentation strengthening method (ESASM), has been reported. Firstly, the rationales of this new method and the resulting changes in internal forces have been studied in details. A numerical optimization analysis was then performed to reach a strengthening design solution based on these results. Secondly, a trial use of this novel strengthening method in a double curved arch bridge has been documented. $A$ numerical simulation was carried out to analyze the internal forces in the bridge both before and after it was strengthened. Results show that internal forces have been favorably redistributed after the bridge has been strengthened by using ESASM. Lastly, the construction method and strengthening effect were discussed.
\end{abstract}

Key word: double curved arch bridge; extrados section augmentation method; structural optimization; low expansion concrete; strengthening; retrofitting 


\section{Introduction}

Double curved arch bridges were one of the most popular arch bridges in the 1950's and 1960's in China. According to statistics data, the total length of double curved arch bridges built during that time has reached $300 \mathrm{~km}$. However, because of the lacking of advanced design methods, under-specified design loads by codes and the inadequate use of steel reinforcement, many double curved arch bridges constructed during that time have an inherent deficiency. This worrying situation has been amplified by the fact that most double curved arch bridges suffer various defects and deterioration due to hostile environmental exposure and a lack of appropriate use and maintenance, such as frequent overloading. As a result, many bridges have presented impending dangers to users [1,2] and the demolition of those problematic bridges has become popular at the beginning of this century. The negative consequence of taking the demolition option is that it does not only lead to a complete disappearing of these bridges, but also causes huge disruptions in traffic and hence often a major economic loss. Engineering inspections have suggested that most double curved arch bridges have various length of working life, and hence they can continue working as long as the causes of the deterioration are indentified and treated accordingly. Therefore, as an alternative to demolishing and rebuilding, the strengthening and retrofitting of existing double curved arch bridge are often considered to have more social and economic benefits.

Researchers and engineers have carried out a large number of research studies on the strengthening and retrofitting of double curved arch bridges. Several key methods have been reported for strengthening purpose, namely,

1) Adding external or internal reinforcement, such as bonding steel plates, mounting steel bars, adding carbon fiber reinforced plastic (CFRP), glass fiber reinforced plastic (GFRP) as internal or external reinforcements [3-5]. The efficiency or economy of these strengthening methods is somewhat low and it will create difficulty and higher cost for the future maintenance work.

2) Tying arch ribs together by adding reinforced concrete slabs, which changes double curved arch bridges into close box arch bridges. This will result into an 
increase in diaphragm stiffness and load bearing area, and ultimately enhance the loading resistance of the bridge [6]. The disadvantage of this method is the need of scaffolding and sometimes requires special construction equipments such as concrete pumps and spraying nozzles if the sprayed concrete is employed. This will inevitably increase the cost and complicate the construction.

3) Altering the structural system, i.e. adding horizontal ties and/or diagonal bracing under arch ribs or spandrel arches. This method is only applicable for small and medium span double curved arch bridges. The added horizontal ties will affect the clear head room below the bridge [7].

4) Arch web augmentation method [8], i.e. enlarging arch ribs by wrapping them with new reinforced concrete. This method needs complicated construction, as it often requires concrete spraying technology or bottom grouting method, which leads to a lower construction efficiency. It is also difficult to control the construction quality if using cast-in-situ concrete [8].

5) Extrados section augmentation method (ESASM) [9-11]. In this method, the extrados section near arch bases will be strengthened by adding new reinforced concrete. This method has been characterized as offering higher efficiency and ease of construction. However, it promotes cracking at the interface between the existing and new concrete due to the differential shrinkage movements, which will compromise the integrity of the strengthened structure. However, if a low-expansion concrete is used as the strengthening material, it will mitigate the stress induced by the differential shrinkage movements and hence will improve the strengthening effect. The inherent slip along the interface will also affect the degree of composite action between the new and old concrete. In order to enhance the composite action, embedding shear reinforcement across the interface can be used.

This paper reports a method of strengthening a double curved arch bridge by using ESAM. It starts with a rationale analysis of using this method and a parametric study is carried out to identify the optimal length of strengthening. Following that, a strengthening project case is presented, in which numerical analysis is carried out to analyze the stress in the bridge both before and after the strengthening is implemented. Results show that critical stress level of the bridge has been considerably reduced after it is strengthened. In addition, a good-practice construction method is recommended to achieve a best strengthening effect. 


\section{Strengthening rationales}

Most early double curved arch bridges were design to be of single span. The primary substructure of this type of bridge is the one-way thrust abutment, often designed with bulky cross-sections of high strength, stiffness and stability. They often have a certain level of safety reserve that can cope with increased traffic loads without a necessity of strengthening. However, it is often the arch ring that becomes problems in responding the modern traffic requirements and constitutes the main reason for strengthening. Theoretical calculation and a number of site investigations have suggested that the vault and skewback are two weakest sections with a high tendency of developing cracks. This has led to the idea of strengthening the section near the skewback by adding more reinforced concrete at the top. This method will have lowest impact on the headroom underneath and the overall appearance of the bridge.

Fig. 1 shows that the dead loads due to the self-weight of the new reinforced concrete are mainly located in the negative zone of the influence line for the bending moment at the vault section. Therefore, these additional loads will reduce the sagging moment as well as the failure occurrence at the vault. In the meanwhile, the increase in stiffness near the skewback section will yield an increased bending moment at the skewback section and a reduced moment at the vault section. This type of internal bending moment shift has constituted the underlying rationales of ESASM.

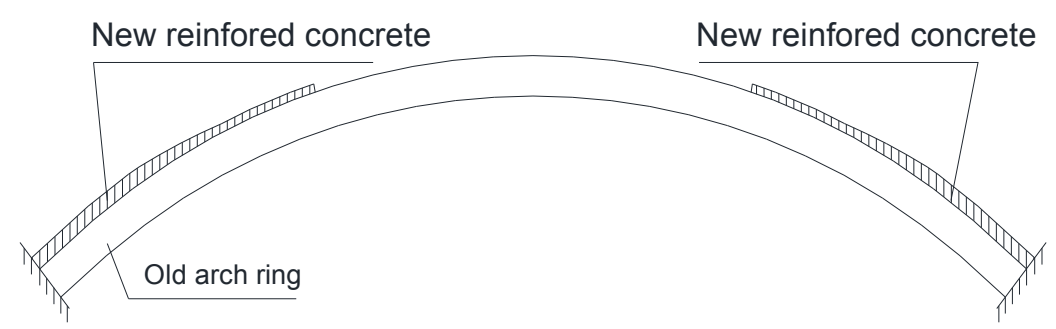

(a) Schematic diagram of ESAM

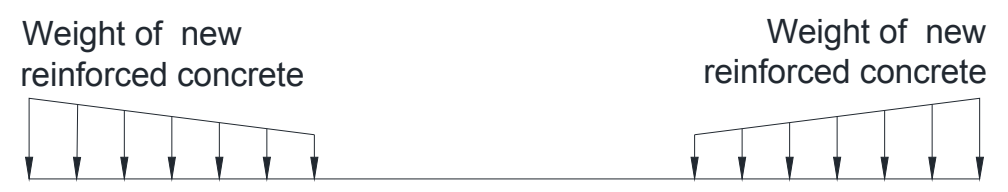

(b) Additional dead load caused by the new reinforced concrete 
(c) Schematic diagram of the influence line for the bending moment at the vault

Fig.1 Rationales of ESASM

\section{Determination on the strengthening length}

A large multitude of arch axis shapes can be seen for the double curved arch bridge, such as arc, catenary, high order polynomial curves, of which the most popular one is the catenary curve. The present study will only consider this type of arch shape. The stress condition of the catenary arch structure is highly dependent on the geometric equation of the arch axis, that is,

$$
\begin{aligned}
& y=\frac{f}{m-1}(\cosh 2 k x / L-1) \\
& k=\ln \left(m+\sqrt{m^{2}-1}\right)
\end{aligned}
$$

$m$ - arch-axis coefficient;

$f$ - rise;

$L$ - span.

As revealed by Eqs. (1) and (2), three design parameters determine the shape of arch, i.e., span, rise-span ratio, arch-axis coefficient. In the following analysis, we will vary the arch span, the rise-span ration and the arch-axis coefficient and examine the effect of these changes on the critical bending moment experienced by the arch bridge.

Considering the idealized calculation model for ESASM as shown in Fig. 2, the arch sections within a horizontal length $l_{s}$ from both skewback sections are strengthened 
with new reinforced concrete. The increase in cross-sectional depth will lead to an increase in the flexural stiffness from $E I$ to $\alpha E I \quad(\alpha>1)$.

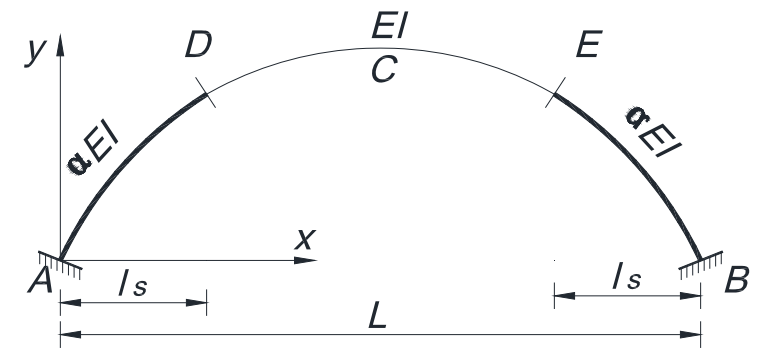

Fig.2 Idealized calculation model

Assume a linear decrease in the new flexural stiffness after the arch section has been enlarged, e.g. $\alpha=3.2$ and 2 at the starting (i.e. at A or B sections) and ending points (i.e. at $D$ or $E$ sections) of each strengthened region, respectively. We chose six strengthening length (i.e. $l_{s}=0.1 L, 0.2 L, 0.3 L, 0.4 L$ and $0.5 L$ ) and examine the bending moment influence line for skewback and vault sections, respectively.

\subsection{Bending moment under different spans (under unit point load)}

Consider the following values of arch span length, i.e. 20, 30, 40 and $50 \mathrm{~m}$, together with a rise-span ratio $1 / 5$, a arch-axis coefficient $m=2.24$ and a moving unit point load; the strengthening length $l_{s}=0,0.1 L, 0.2 L, 0.3 L, 0.4 L$ and $0.5 L$. The bending moment of the arch structure before and after strengthening are calculated for each case by using a finite element analysis.

Fig. 3 show the bending moment influence lines of the vault section before and after the strengthening with various strengthening length for arch spans $L 20,30,40,50 \mathrm{~m}$ respectively. The insets in Fig. 3 show a close view of these curves near their positive peak points. Results show a common trend for the change of the influence line for the vault moment under different arch span length. Fig. 3 also reveals that both negative and positive moments will reduce when $l_{s}$ increases up to $0.5 \mathrm{~L}$. But when $l_{s}=0.5 \mathrm{~L}$ (fully 
strengthened), they will revert close to the case of $l_{s}=0$, i.e. the un-strengthened case. If the entire arch has been strengthened uniformly, the influence line of vault moment before and after strengthening will be identical.

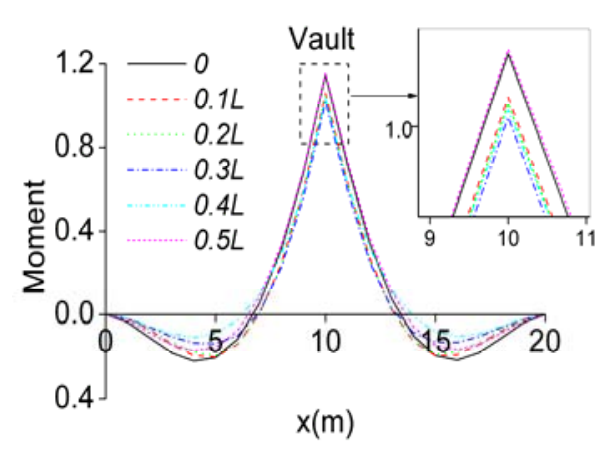

(a) $L=20 \mathrm{~m}$

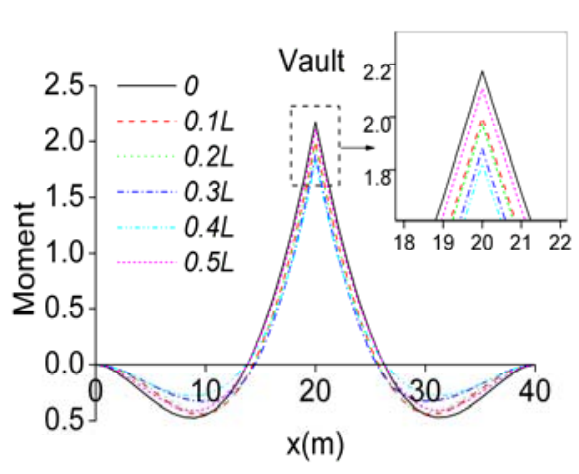

(c) $L=40 \mathrm{~m}$

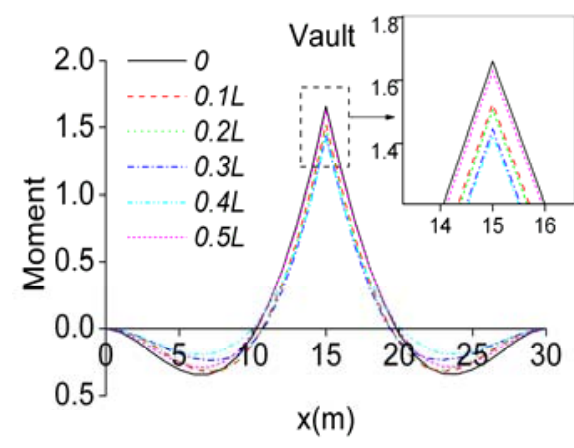

(b) $L=30 \mathrm{~m}$

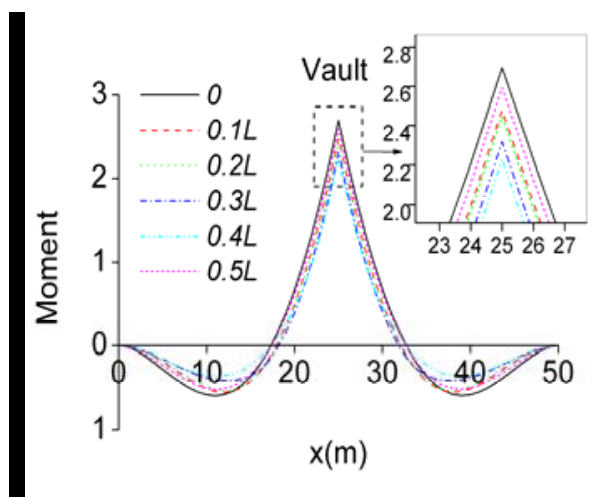

(d) $L=50 \mathrm{~m}$

Fig. 3 Influence lines of vault moment under arch spans of $20,30,40,50 \mathrm{~m}$

Fig. 4 shows that the strengthening will increase the skewback moment. The maximum negative value for the skewback moment is discovered at $L_{s}=0.1 L$, and the increase in strengthening length will result into a reduction in the negative peak values. The positive sections of the curves for the five strengthening lengths are quite similar for $L s$ ranging between 0.1 and $0.3 L$, in which $L s=0.2 L$ shows a slightly higher peak value. The curves for $L s=0.5 \mathrm{~L}$ are close to the un-strengthend case; in particular, in the positive zone, the curves for $L s=0$ and $L s=0.5 L$ are almost coincident. 


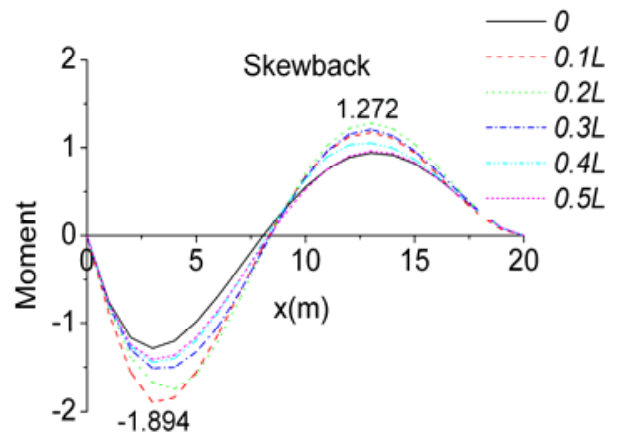

(a) $L=20 \mathrm{~m}$

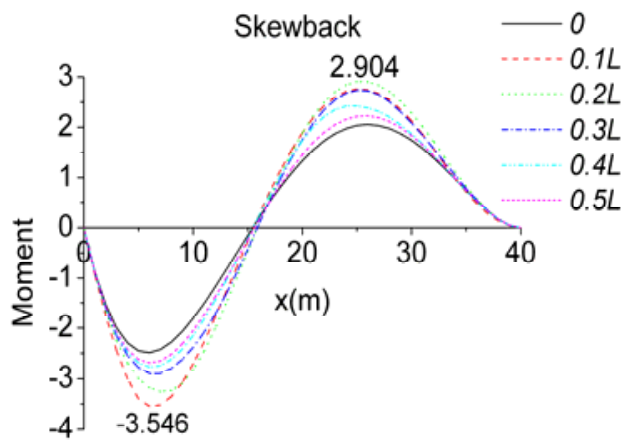

(c) $L=40 \mathrm{~m}$

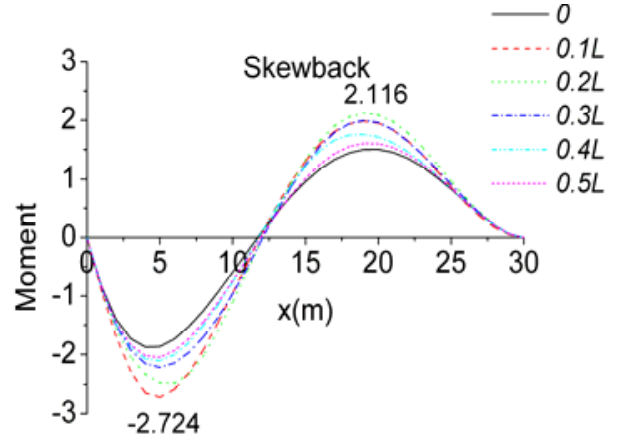

(b) $L=30 \mathrm{~m}$

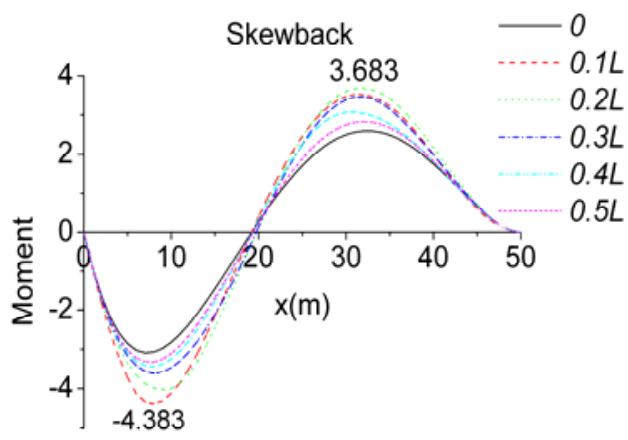

(d) $L=50 \mathrm{~m}$

Fig.4 Influence lines of skewback moment under arch spans of 20, 30, 40 and 50m

\subsection{Bending moment under different rise-span ratios}

Consider $L=40 \mathrm{~m}, m=2.24$, four different values for the rise-span ratio, i.e. $1 / 4,1 / 5,1 / 6,1 / 7$ and the strengthening length $L_{s}=0,0.1 \mathrm{~L}, 0.2 \mathrm{~L}, 0.3 \mathrm{~L}, 0.4 \mathrm{~L}$ and $0.5 \mathrm{~L}$ respectively, the bending moment of the arch structure before and after strengthening are calculated by using FEA.

The influence lines of vault moment are presented in Fig. 5. It is interesting to find that under various rise-span ratios, the trend of results is quite consistent. In each rise-span ratio case, the effect of strengthening length on the peak values of the influence line is similar to the case of varying the bridge span. 


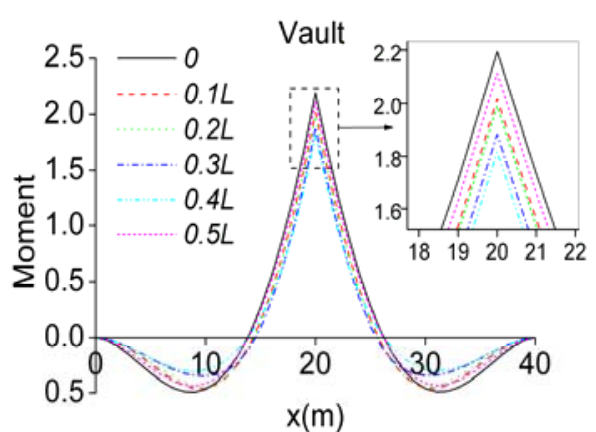

(a) $f / L=1 / 4$

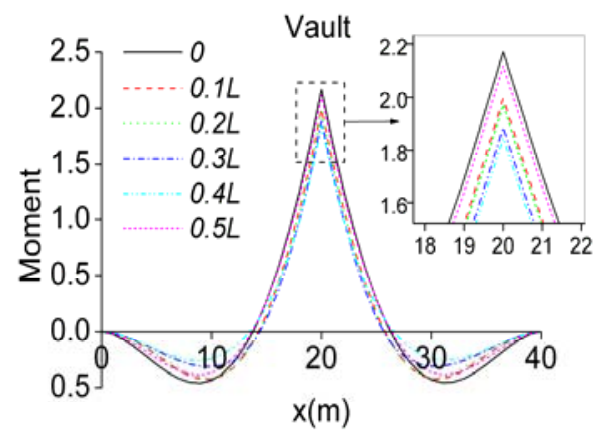

(c) $f / L=1 / 6$

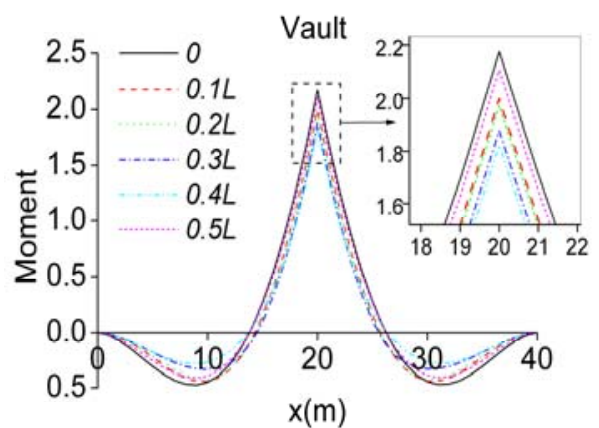

(b) $f / L=1 / 5$

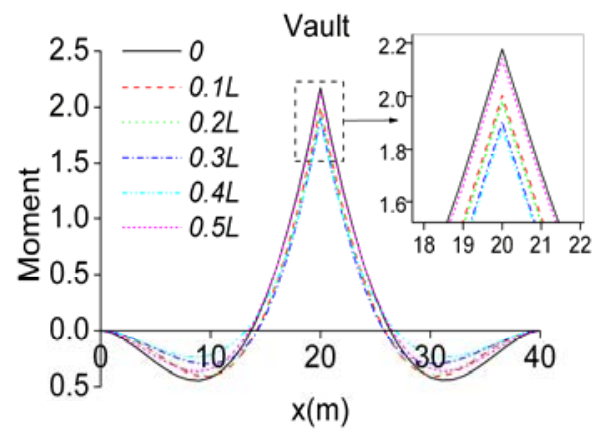

(d) $f / L=1 / 7$

Fig.5 Influence lines of vault moment under the rise-span ratio of $1 / 4,1 / 5,1 / 6,1 / 7$

The influence lines of skewback moment are also calculated and presented in Fig. 6. The results also show a consistent trend on the influence of the strengthening length under different rise-span ratios. When $L s=0.1-0.3 L$, the effect of strengthening length on the negative moment is more distinct than the positive one. The negative moment is often more critical in the design practice. $L s=0.1 L$ leads to a maximum negative peak value that is $45 \%$ higher than the un-strengthened case, and the increase ratio for positive peak value is only around $35 \%$. 


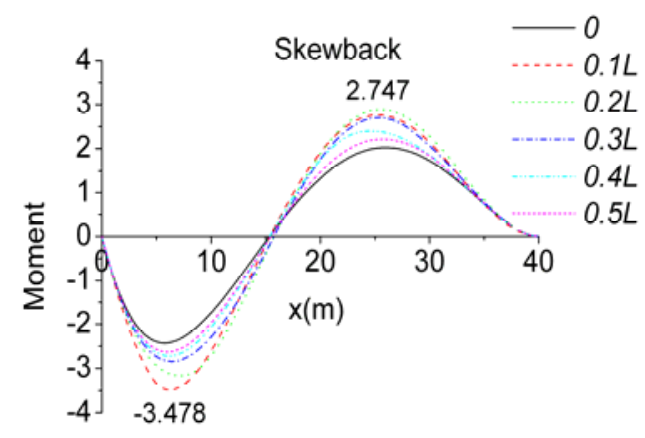

(a) $f / L=1 / 4$

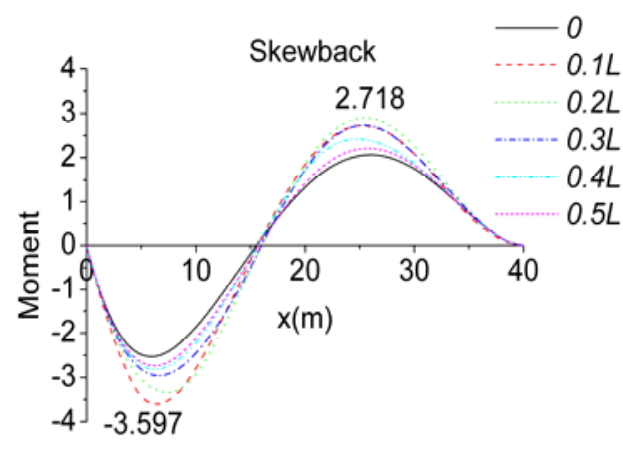

(c) $f / L=1 / 6$

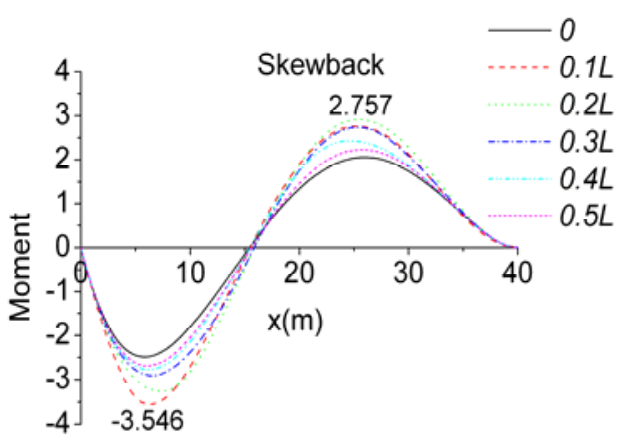

(b) $f / L=1 / 5$

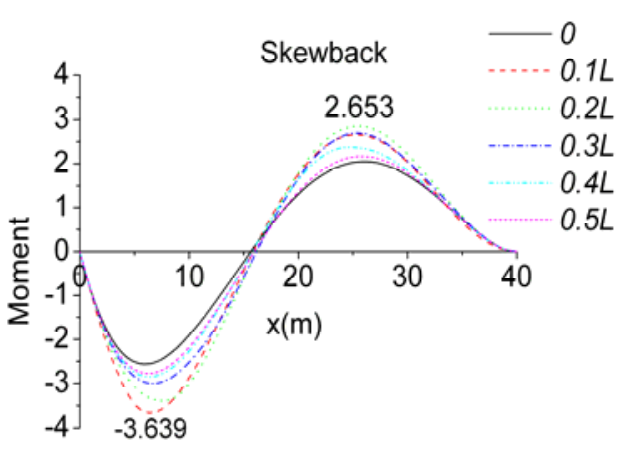

(d) $f / L=1 / 7$

Fig.6 Skewback moment influence lines under the rise-span ratio of $1 / 4,1 / 5,1 / 6,1 / 7$

\subsection{Bending moment under different arch-axis coefficients}

The strengthening construction for bridges with low arch-axis ratio often does not require props or can have an early removal of props. Therefore only low arch-axis ration values are selected in this study. Consider $m=1.543,1.988,2.514,3.142$, respectively [12]; rise-span ratio $=1 / 5, L=40 \mathrm{~m}$; the length of reinforcement $l_{s}=$ $0,0.1 L, 0.2 L, 0.3 L, 0.4 L$ and $0.5 L$. The bending moment of the arch structure before and after strengthening are calculated and presented for discussion.

The vault moment influence lines are shown in Fig. 7. Similar observations can be made to the previous cases when the span and rise-span ratio are varied. The rate of reduction in vault moment after strengthening is by large limited, e.g. about $20 \%$ for 
the positive peak and $30 \%$ for the negative peak. The peak values of the positive peak moment are almost four times as high as those of negative moment.

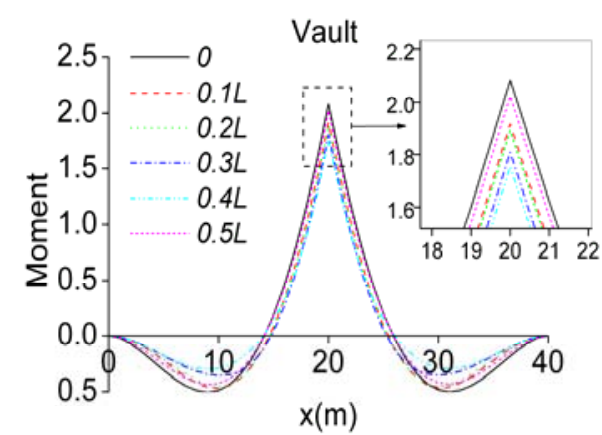

(a) $m=1.543$

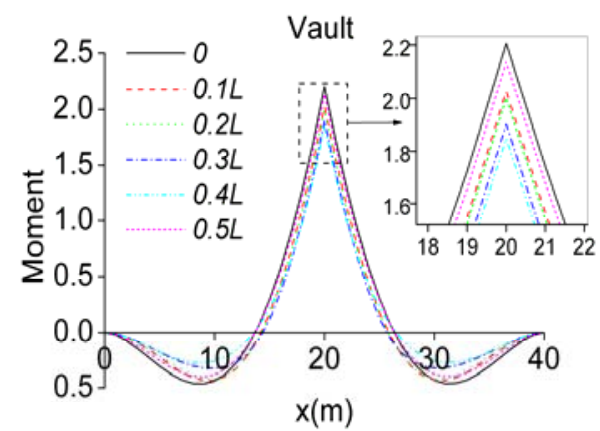

(c) $m=2.514$

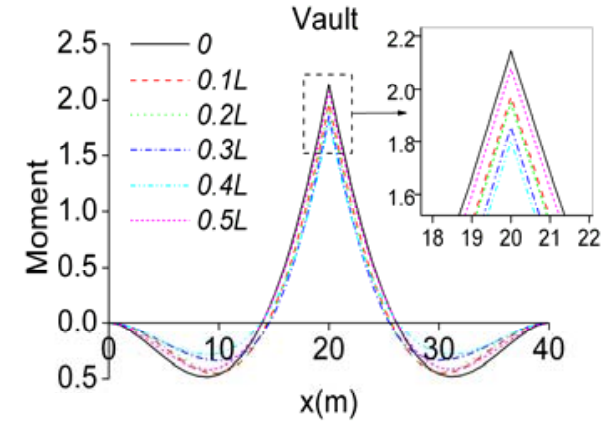

(b) $m=1.988$

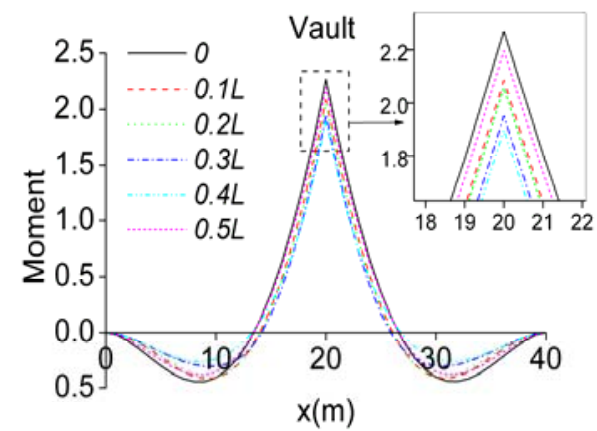

(d) $m=3.142$

Fig.7 Vault moment influence lines under the arch-axis coefficient of $1.543,1.988$,

The results of skewback moment influence lines are presented in Fig. 8, which show a very similar trend to Figs. 4 and 6.
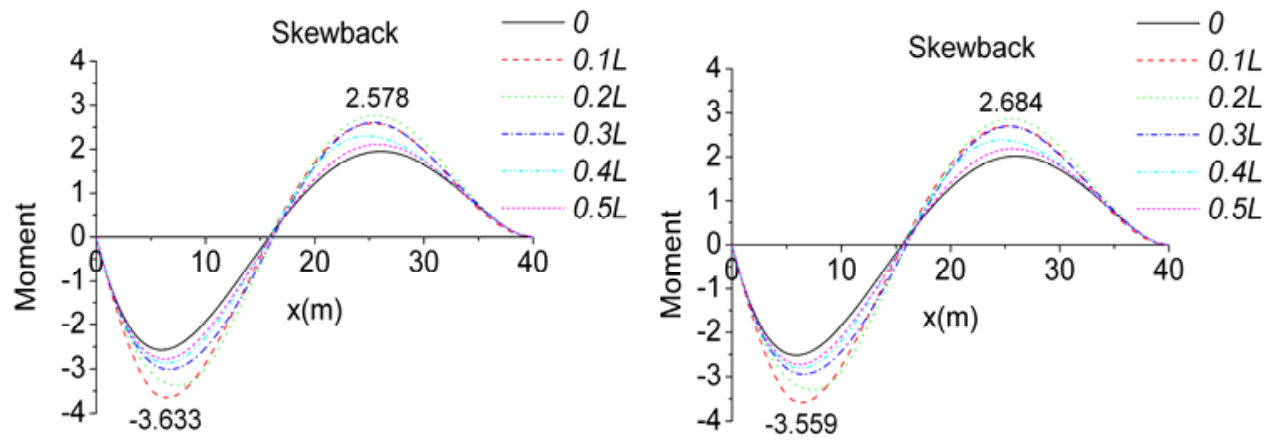
(a) $m=1.543$

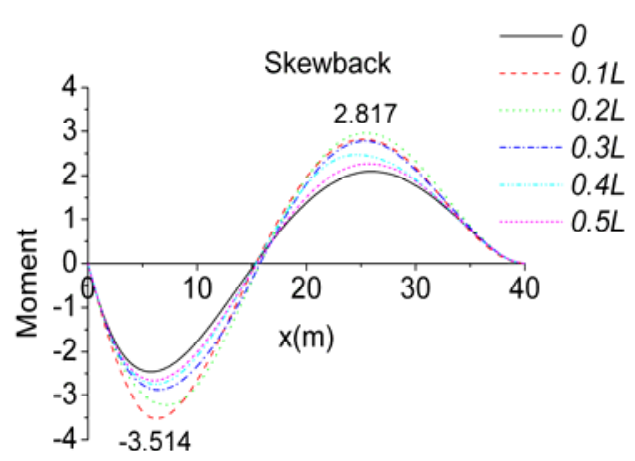

(c) $m=2.514$ (b) $m=1.988$

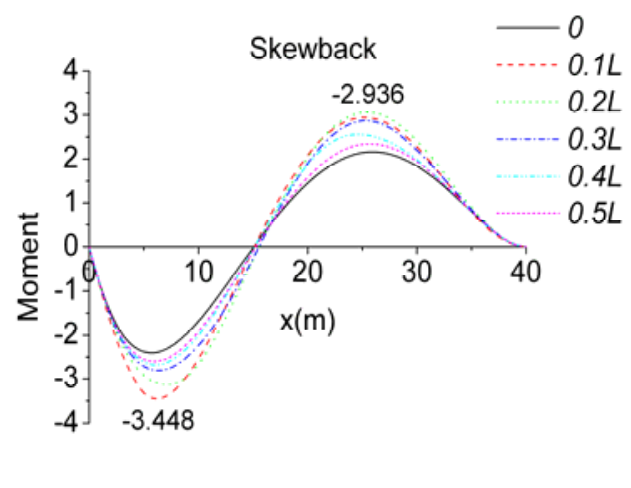

(d) $m=3.142$

Fig. 8 Skewback moment influence lines under the arch-axis coefficient of 1.543,

$1.988,2.514,3.142$

\subsection{Discussions of parametric analysis}

The above comparisons of the internal moment in the double curved arch bridges with different design parameters lead to the following observations:

1) For bridges with a wide range of design parameters on span length, rise-span ratio, arch-axis coefficient, the effect of strengthening length on the bending moments in the skewback and vault sections is similar.

2) The critical moments at the vault sections will decline while the strengthening length is increased. The positive moment at the vault section that remains the same after strengthening will decrease while the strengthening length increases. When the entire arch span is strengthened, the moment at the vault section will suddenly return to the same value before strengthening. It is worth noting that by then, the vault section has also been strengthened and hence the stress induced by the moment is effectively reduced. The moment at the skewback section reaches the maximum value when the strengthening length is $10 \%$ of the bridge span and, after that, starts to decrease while increasing the strengthening length. Once the entire bridge span is strengthened, the moment will drop to the same value as before strengthening. In general, the effect of increasing the strengthening length has a positive effect on the critical 
design moments in the arch structure.

3) The maximum positive and negative moments at the vault and the skewback sections for the bridge span 20, 30, 40 and 50m (span-rise ratio is $1 / 5, m=2.24$ ) are presented for two strengthening design cases, which, respectively represent a short $\left(l_{s}=0.1 L\right)$ and long $\left(l_{s}=0.4 L\right)$ strengthening length (see Table 1).

Table 1 The critical moment at vault and skewback sections

\begin{tabular}{cccccccc}
\hline \multirow{2}{*}{ Position } & \multirow{4}{*}{ Span } & \multicolumn{3}{c}{ Max positive moment } & \multicolumn{3}{c}{ Max negative moment } \\
& & $0.1 L(\mathrm{~A})$ & $0.4 \mathrm{~L}(\mathrm{~B})$ & $(\mathrm{B}-\mathrm{A}) / \mathrm{A}$ & $0.1 \mathrm{~L}(\mathrm{~A})$ & $0.4 \mathrm{~L}(\mathrm{~B})$ & $(\mathrm{B}-\mathrm{A}) / \mathrm{A}$ \\
\hline \multirow{6}{*}{ Vault } & $20 \mathrm{~m}$ & 1.060 & 1.034 & $-2.5 \%$ & - & - & - \\
& $30 \mathrm{~m}$ & 1.524 & 1.425 & $-6.5 \%$ & - & - & - \\
& $40 \mathrm{~m}$ & 1.999 & 1.824 & $-8.8 \%$ & - & - & - \\
& $50 \mathrm{~m}$ & 2.479 & 2.229 & $-10.1 \%$ & - & - & - \\
& $20 \mathrm{~m}$ & - & - & - & -1.894 & -1.444 & $-23.8 \%$ \\
\multirow{6}{*}{ Skewback } & $30 \mathrm{~m}$ & - & - & - & -2.724 & -2.104 & $-22.8 \%$ \\
& $40 \mathrm{~m}$ & - & - & - & -3.546 & -2.772 & $-21.8 \%$ \\
& $50 \mathrm{~m}$ & - & - & - & -4.383 & -3.441 & $-21.5 \%$ \\
\hline
\end{tabular}

As is revealed by Table1, the positive moment at the vault section decreases between $2.5 \%$ and $10.1 \%$, when the strengthening length increase from $0.1 \mathrm{~L}$ to $0.4 \mathrm{~L}$. The negative moment at the skew back section decreases between $21.5 \%$ and $23.8 \%$. Therefore, in the context of the reduction in critical moment, a large reinforcement length is recommended; however, the consideration of economical efficiency usually does not support the design choice of choosing $l_{s}=0.4 L$. A trade-off between the strengthening length and strengthening section will be found to maximize the economical efficiency.

\section{Project case}

Bai-guo-shu-ping bridge is a double curved arch bridge on S307 provincial road located in Xiangyang city Hubei province. The bridge was built in 1977, with a length 
$57 \mathrm{~m}$, width $8 \mathrm{~m}$ and the original design load was $15 \mathrm{t}$ for vehicle, and $80 \mathrm{t}$ for trailer. The bridge is a catenary double curved arch bridge with a uniform cross section. The clear span length is $40 \mathrm{~m}$; the arch axis coefficient is 2.24 ; and the rise-span ratio is 1/7. The substructure of the bridge is a gravity abutment with an expanded foundation. The main arch ring consists of five arch ribs and four complete and two half arches with a depth of $1.05 \mathrm{~m}$. The structure of the arch bridge is illustrated in Fig. 9.

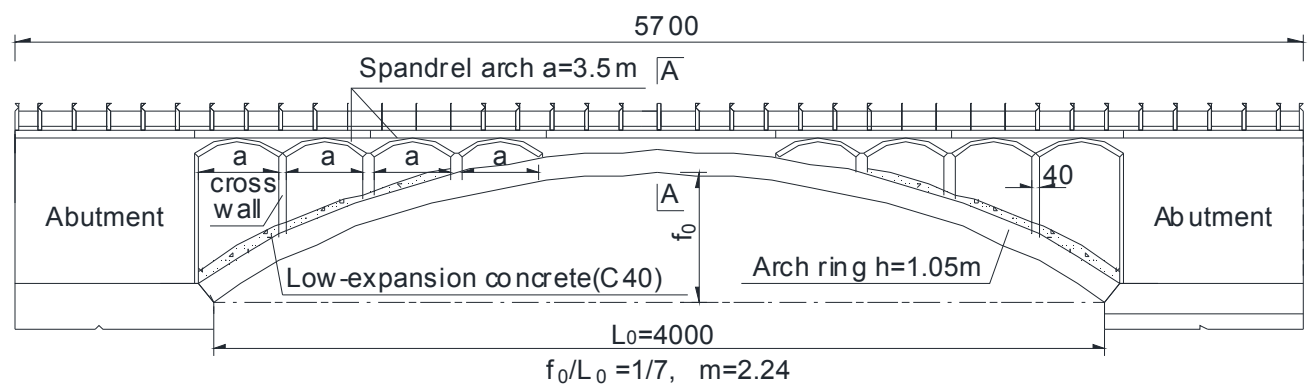

(a) Elevation view

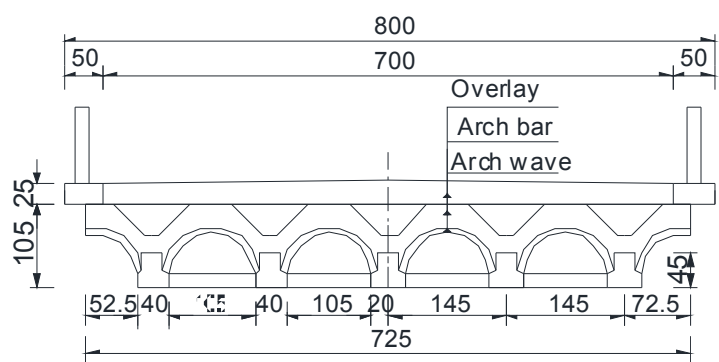

(b) A-A cross section

Fig. 9 Overall structure of the arch bridge (in $\mathrm{cm}$ )

The main defect of the primary arch ring and the spandrel arch include cracks in the main arch ring, exposed reinforcing steel, corrosion of steel bars, alkaline corrosion on the surface ( see Figs.10-12) and damages and delamination in spandrel arches (see Fig.13). 


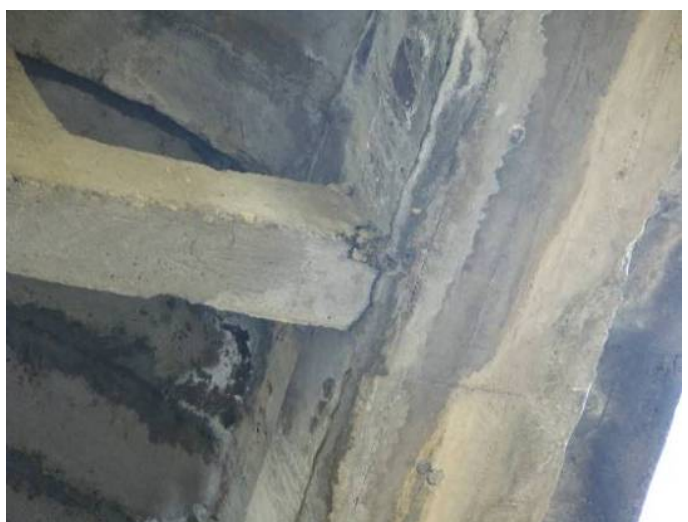

Fig.10 Corrosion in the main arch ring

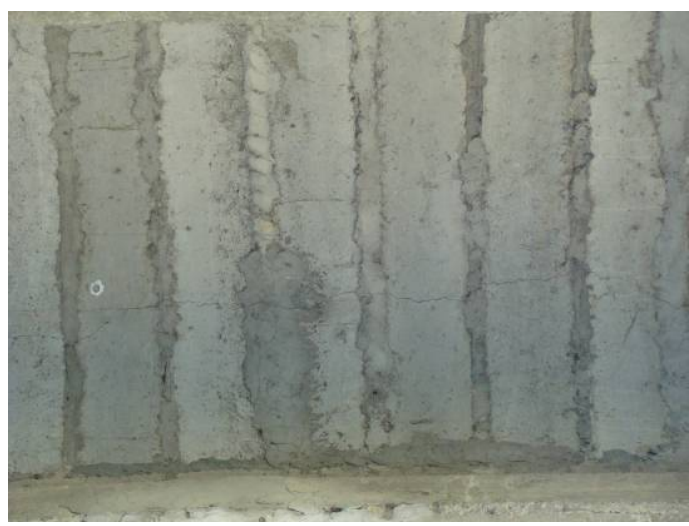

Fig.12 Major cracks between adjacent arches

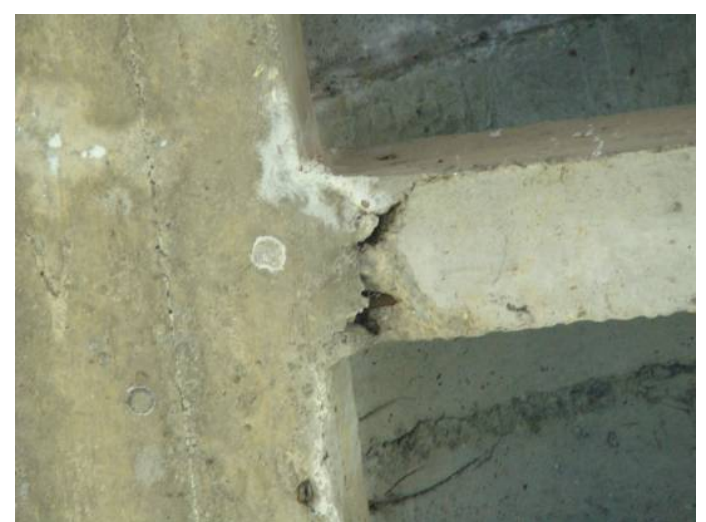

Fig.11 Exposed reinforcing steel in the diaphragm beam

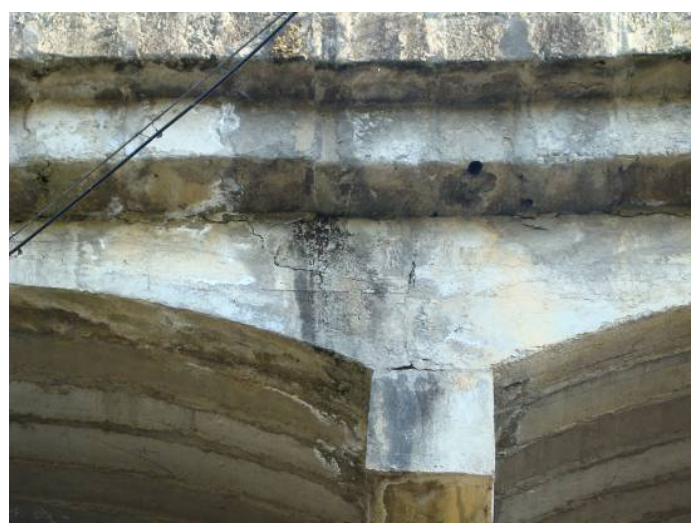

Fig.13 Damage in the spandrel arch

These defects in the main arch ring of this bridge have led to a huge deficiency in meeting the current loading requirement due to an increasing traffic. Strengthening work had to be carried out immediately. ESASM has been selected in this project, i.e. adding low-expansion concrete on the extrados from the skewback inwards, with a height $45 \mathrm{~cm}$ at the skewback and $25 \mathrm{~cm}$ at the first cross wall from the vault section. A curved variation in the depth of new concrete was chosen between these two locations.

In order to create a sound bond between the old and new concrete, the following treatment has been taken: (i) the extrados was thoroughly cleaned by using steel brushes followed by water jet cleaning; after the surface dried, it was then manually roughened; (ii) embedding vertical rebars into the old concrete; the exposed rebars 
were tied to the new steel reinforcement. The vertical and main reinforcement for the new concrete is shown in Fig.14.

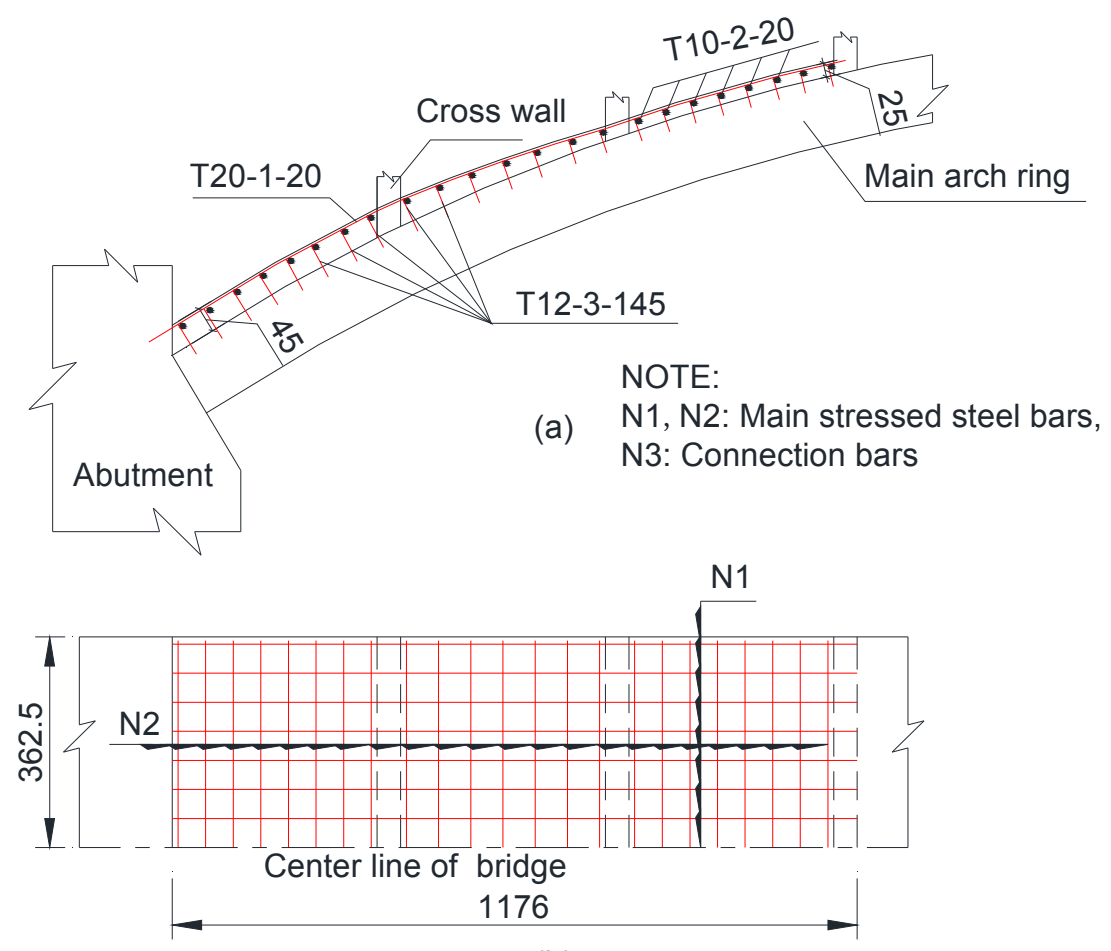

(b)

(a) Elevation view of reinforcement detail

(b) Plan view of the reinforcement detail

Fig.14 Reinforcement arrangement for strengthening

A 3-D grillage finite element model was developed to analyze the bridge before and after it was strengthened. Five curved longitudinal beams were created for main arch ribs, which were connected by cross beams. Longitudinal beams were meshed into beam element every meter. The cross wall and spandrel arch elements were also created in the transverse direction. There were in total 455 beam elements in the model. The material of the main arch ring and cross wall was C25. Fixed boundary conditions were adopted. The 3-D grillage model is shown in Fig.15. 


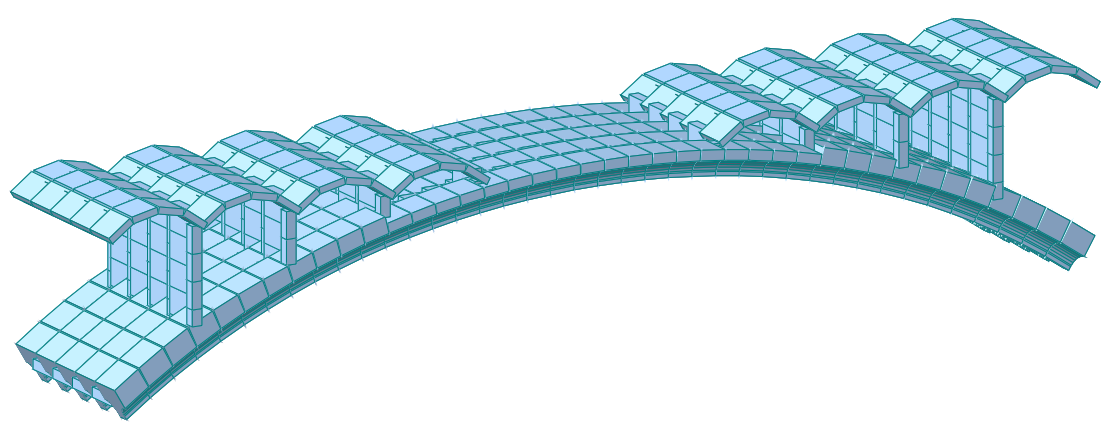

Fig.15 3-D grillage model of the strengthened double curved arch bridge

The loading category is highway load class II [13]. Both the symmetric and asymmetric loads were applied on the model to analyze the internal forces of the bridge before and after strengthening. The loading arrangements were shown in Fig.16. The max. positive and negative moments of the arch structure before and after strengthening are analyzed for both asymmetric and symmetric loads and they are presented in Figs. 17 and 18.

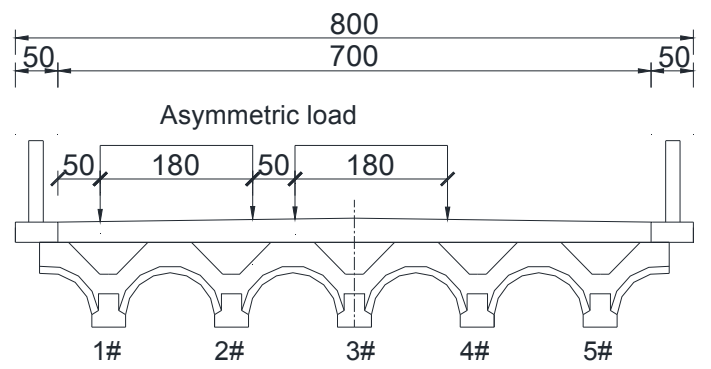

(a) Asymmetric load method

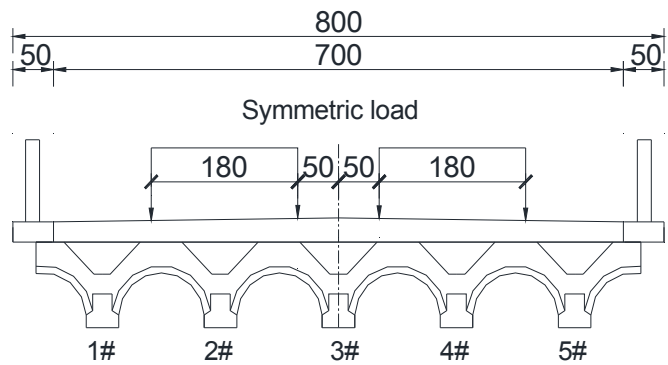

(b) Symmetric load method

Fig.16 Loading arrangement

The maximum positive moments under the asymmetric load at vault and skewback sections before and after strengthening are shown in Fig.17(a). It can be seen that under the asymmetric load, five arch ribs experience same level positive moment at the skewback section; whereas at the vault section, the middle rib carries the highest bending moment, nearly twice as high as the adjacent one. The maximum positive moment at the skewback increases about $49.7 \% \sim 50.2 \%$ compared to the original moment. The maximum positive vault moment decrease about 5.9\% 18.9\% after strengthening. 
The maximum negative moments of vault and skewback under the asymmetric load before and after strengthening are shown in Fig.17 (b). The negative moments in each rib are nearly uniform for the vault section. Results shows that the maximum negative moment at the skewback section increases about $45.5 \% \sim 49.7 \%$ after strengthening; and the maximum negative moment at the vault section decreases about $12.8 \% \sim 15.4 \%$ after strengthening.
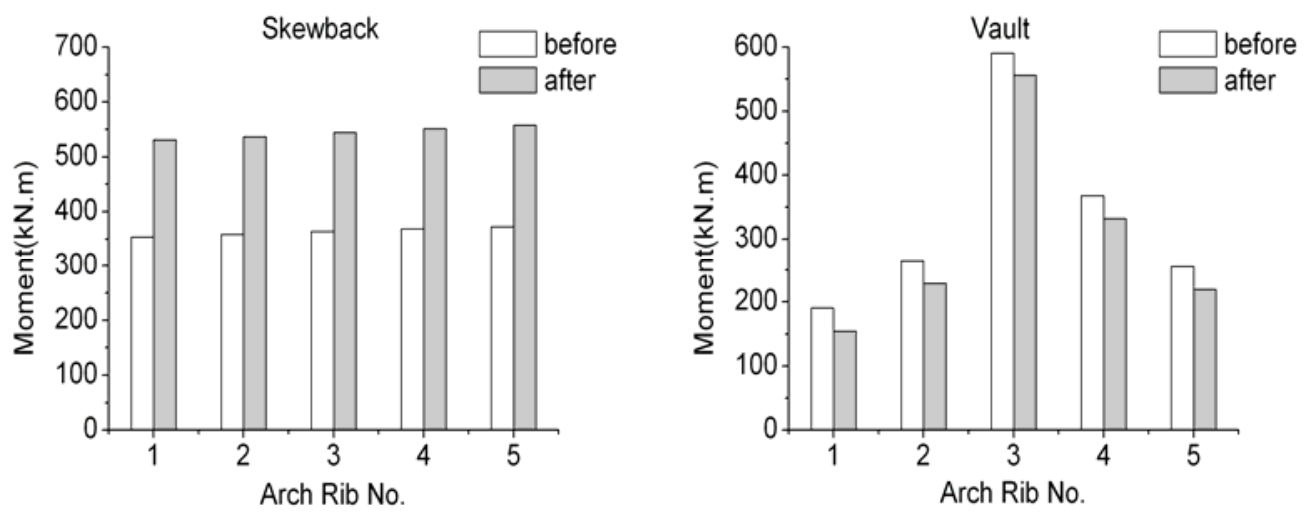

(a) Max. positive moments
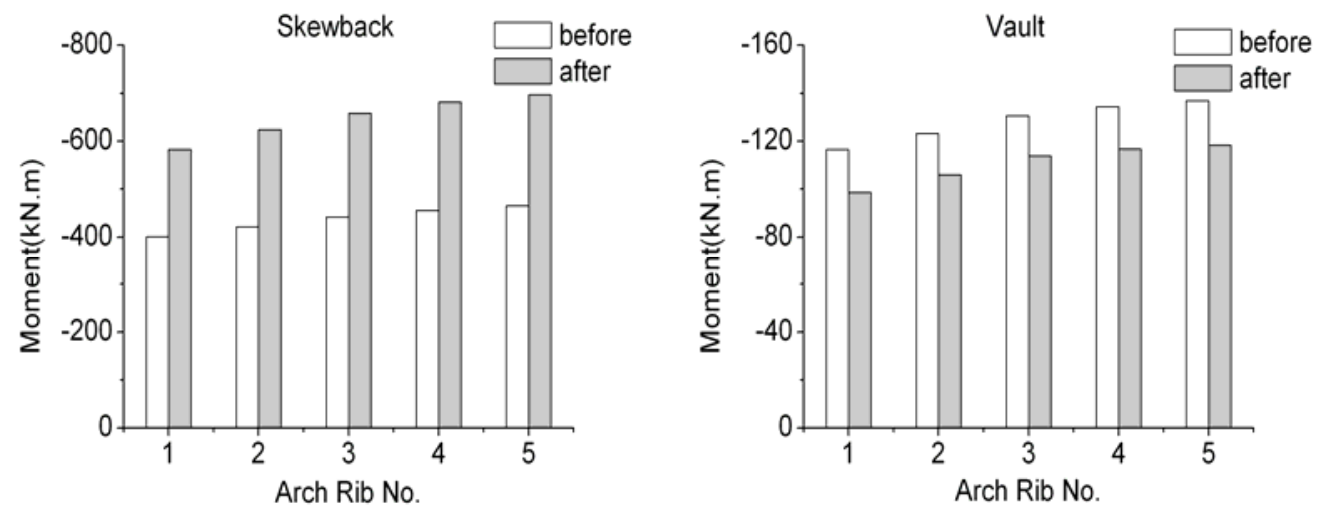

(b) Max. negative moments

Fig.17 Max. moments at skewback and vault sections under asymmetric load

The maximum positive moments of vault and skewback under the symmetric load before and after strengening are shown in Fig.18 (a). It can be seen that under the symmetric load, the moment experienced in each rib are uniform except the sagging moment at the vault section, where the mid-rib carries the highest moment. The maximum positive moment of the skewback increase about $49.8 \%$ compared to the 
unstrengthened case; the maximum positive moment at the vault decrease about $5.9 \% \sim 16.2 \%$.

The maximum negative moment of vault and skewback under the symmetric load before and after strengthening are shown in Fig. 18(b). It can be seen that the increase of the maximum negative moment at the skewback is about $47.7 \% \sim 48.9 \%$; while that at the vault decreases about $12.8 \% \sim 14.5 \%$ to the original moment.
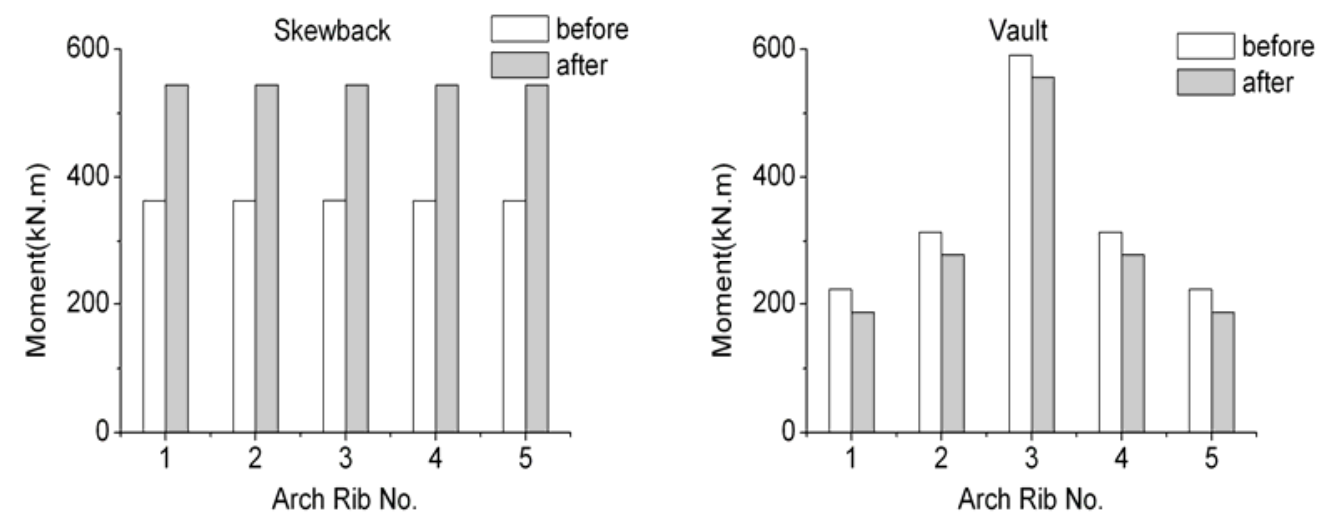

(a) Max. positive moments
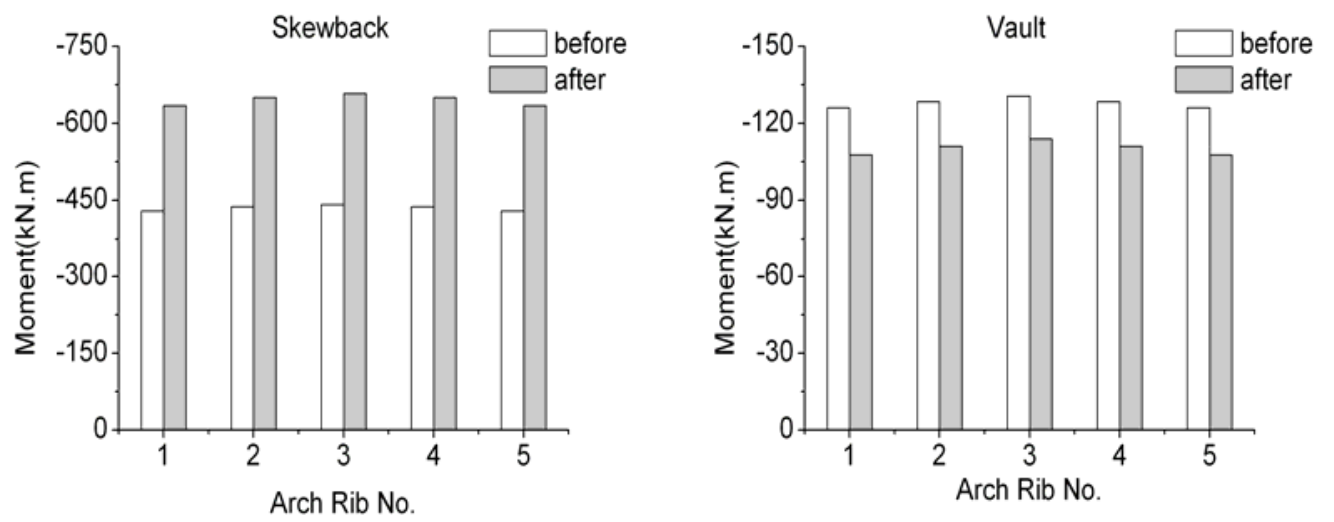

(b) Max. negative moments

Fig.18 Max. moments at vault and skewback sections under the symmetric load

\section{Construction method and strengthening effect}


The construction method of ESASM used in this project was relatively easy. It did not need scaffolding or formwork. The disruption to the traffic on the bridge was minimal. The main construction method was as follows:

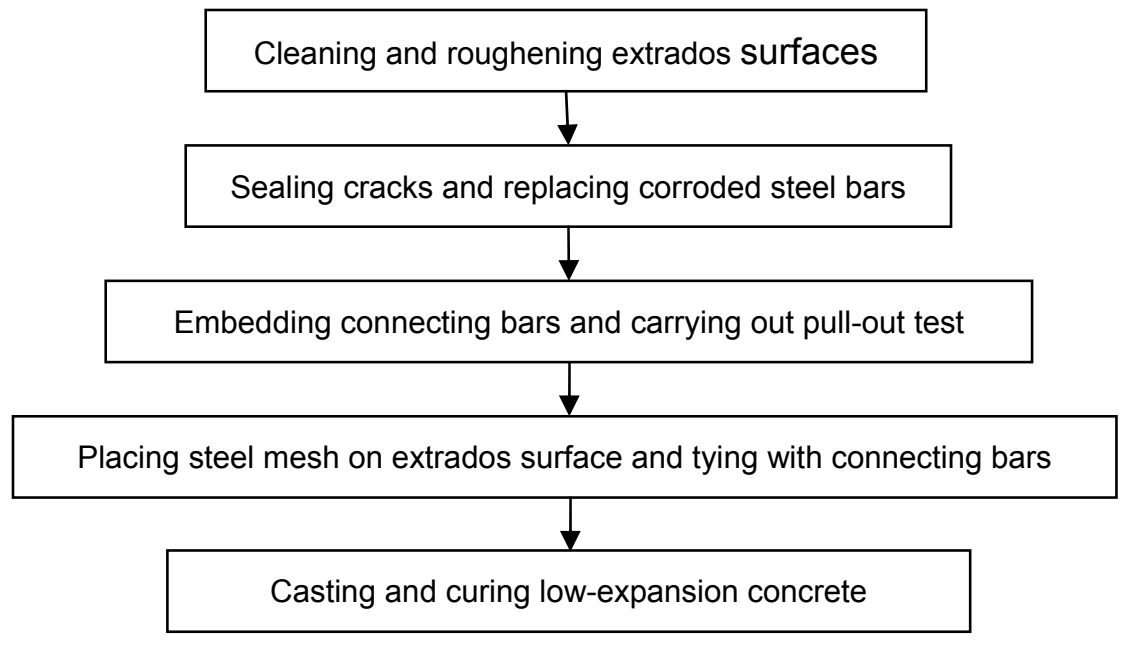

Fig.19 Construction method of ESASM

The strengthening construction was completed in 2008 and has been inspected in the past 4 years. The bridge has been in operation under satisfactory conditions without large cracks. The effectiveness of ESASM has been well demonstrated.

\section{Conclusions}

Various strengthening methods for double curved arch bridges have been well exploited. The extrados section augmentation strengthening method (ESASM) has displayed obvious advantages compared to other methods. This paper reports a parametric analysis to examine the effect of strengthening length on the internal bending moment in arch structures with various design parameters. The common trend of the moment variation under differing strengthening length has been discussed, namely, increasing strengthening length will reduce the critical moments at the vault section. However, in practice, the determination of strengthening length will be a trade-off between the cross-sectional area and length, from which a high cost effectiveness can be achieved. The construction method and the effect of adopting 
ESASM have been demonstrated via a case project. It leads to the following conclusions:

1) Adding reinforced low-expansion concrete in ESASM on the extrados will stiffen the section near the skewback and hence change the stiffness distribution along the arch structure, leading to a reduction in the vault moment. Therefore, the design moment in the unstrengthened vault section will be reduced and, as a result, the safety reserve will be enhanced. In the meanwhile, the negative moment at the skewback section has been increased, which can be dealt with by adding more reinforcement.

2) Along with the increases of reinforcement length $l_{s}$, the positive moment of the vault and the negative moment of the skewback decrease too. The increasing of the strengthening length has a positive influence on the moment of the vault and skewback.

3) The strengthening project of Bai-guo-shu-ping bridge has demonstrate that after strengthening, the vault moment has been reduced by $5.9 \%$ to $16.2 \%$ for the symmetric load case, and by $12.8 \%$ to $15.4 \%$ for the asymmetric loadg case; whereas the skewback moment has been increased by $47.7 \%$ to $48.9 \%$ for the symmetric load case, and by $49.7 \%$ to $50.2 \%$ for the asymmetric load case. The loading capacity of the main ring arch has been enhanced significantly, so has the integrity. Four year's frequent inspection has shown that the current method is safe, easy to construction and effective.

4) ESASM can also be used in strengthening the box-beam arch bridges, framed arch bridges and open-spandrel stone arch bridge.

\section{Acknowledgements}

Project supported by the Natural Science Foundation of Hubei Province (2009CDB095) and Fundamental Research Funds for the Central Universities (2010-IV-062). 


\section{References}

[1] ALI FADHIL NASER,WANG ZONGLIN. Damage Investigation, Strengthening, and Repair of Jilin Highway Double-Curved Arch Concrete Bridge in China[J]. Procedia Engineering 14 (2011) 2294-2300.

[2] A. Thavalingam, N. Bicanic, J.I. Robinson, D.A. Ponniah. Computational framework for discontinuous modeling of masonry arch bridges[J]. Computers and Structures 79 (2001) 1821-1830.

[3] Caporale, L. Feo, R. Luciano. Limit analysis of FRP strengthened masonry arches via nonlinear and linear programming[J]. Composites: Part B 43 (2012) 439-446-

[4] Laura De Lorenzis, Rossana Dimitri, Antonio La Tegola. Reduction of the lateral thrust of masonry arches and vaults with FRP composites[J]. Construction and Building Materials 21 (2007) 1415-1430.

[5] Jun He, Yuqing Liu, Airong Chen, Liang Dai. Experimental investigation of movable hybrid GFRP and concrete bridge deck[J]. Construction and Building Materials, Volume 26,Issue 1,January 2012,49-64.

[6] Yuan Li-hua, Yu Yi. Research of changing the double arch into box arch bridge by adding concrete plane. Highways \& Automotive Applications,2011,(3):171-172.(In Chinese)

[7] Yuan Haiqing, Wang Ji, Lu Zhe'an. The Method of Consolidating Double Curverd Arch Bridge by Shortening Span and its Application in Engineering[J]. JOURNAL OF WUHAN UNIVERSITY OF TECHNOLOGY,2002,(6):58-61. (In Chinese)

[8] He Qin. Application of Sectional Area Variable Arch in Dangerous Road Bridge Strengthening[J].Technology of Highway and Transport,2006,(4):109-111. (In Chinese)

[9] Kang Jun-tao, Wang Guo-ding, Gao Rong-xiong, E De-jun. Strengthening of Bridges for Special Loads to Pass through[J]. World Bridges,2004,(2):62-64. (In Chinese) 
[10] ZHOU Shui-xing, ZHANG Min, ZHOU Ying-quan. Pull Calculation of Embedded Bars for Reinforced Arch Bridge with Enlarged Cross-Section[J].Highway,2005,(8):13-15 .(In Chinese)

[11] ZHOU Zhi-xiang, HE Peng, ZHAO Chan-hui. Research on reinforcing and repairing of a long-span double curved arch bridge[J].Journal of Chongqing Jiaotong University,2004,(3):1-4. (In Chinese)

[12] Gu Anbang, Sun Guozhu. Bridge Design Manual: Arch Bridge(the first volume) [M]. Beijing: People's traffic press, July 2000. (In Chinese)

[13] JTG D60-2004, The Chinese industry standards: General Code for Design of Highway Bridges and culverts[S]. Beijing: People's traffic press, October 2004. (In Chinese) 